\title{
Author Proof
}

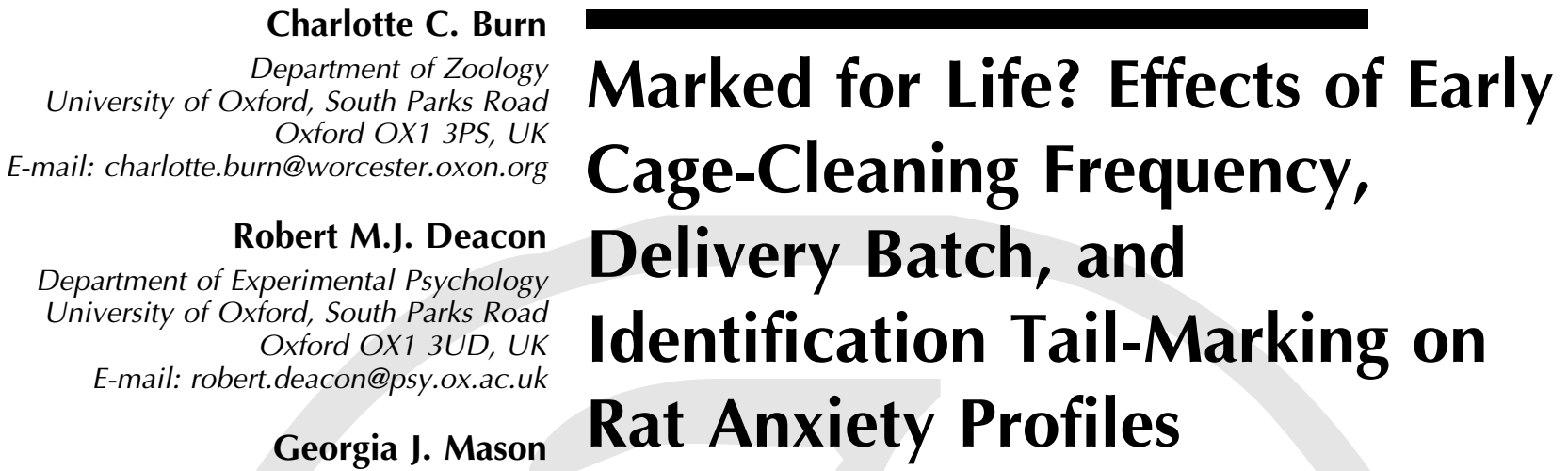

Department of Animal \& Poultry Science University of Guelph Ontario N1G 2W1, Canada E-mail: gmason@uoguelph.ca

\begin{abstract}
Daily handling of preweanling rats reduces their adult anxiety. Even routine cage-cleaning, involving handling, reduces adult anxiety compared with controls. Cage-cleaning regimes differ between animal breeders, potentially affecting rodent anxiety and experimental results. Here, 92 adult male rats given different cage-cleaning rates as pups, were compared on plus-maze, hyponeophagia, corticosterone, and handling tests. They were pair-housed and half were tailmarked for identification. Anxiety/stress profiles were unaffected by cage-cleaning frequency, suggesting that commercial-typical differences in husbandry contribute little variance to adult rat behavior. However, delivery batch affected some elevated plus-maze measures. Also, tail-marked rats spent three times longer on the plusmaze open arms than their unmarked cagemates, suggesting reduced anxiety, yet paradoxically they showed greater chromodacryorrhoea responses to handling, implying increased aversion to human contact. A follow-up study showed that rats avoided the odor released from the marker pen used. Thus, apparently trivial aspects of procedure can greatly affect experimental results.

(c) 2008 Wiley Periodicals, Inc. Dev Psychobiol 9999: 1-12, 2008.
\end{abstract}

Keywords: husbandry; anxiety; Rattus norvegicus; early experience; elevated plus-maze; standardization

\section{INTRODUCTION}

Since the late 1950s it has been known that early handling (EH)-the brief daily handling of neonatal rodent pups before weaning-causes lasting reductions in anxiety throughout rodents' lives (Levine, 1958). This is reflected in behavioral, endocrine, physiological, immunological, and neuro-histochemical changes (reviewed in FernandezTeruel et al., 2002; Meaney, Mitchell, Aitken, \& Bhatnagar,

Received 19 October 2006; Accepted 27 September 2007

Correspondence to: C.C. Burn

Contract grant sponsor: UK Home Office

Contract grant sponsor: BBSRC (UK) and NSERC (Canada)

Published online in Wiley InterScience

(www.interscience.wiley.com). DOI 10.1002/dev.20279

(c) 2008 Wiley Periodicals, Inc.
1991; Pryce \& Feldon, 2003). EH usually involves $15 \mathrm{~min}$ separation from the mother, but anxiolytic effects have also been observed after as little as 3 min handling (e.g., Denenberg \& Smith, 1963; Denenberg \& Whimbey, 1963; von Hoersten, Dimitrijevic, Markovic, \& Jankovic, 1993). EH effects might be mediated by early stimulation per se, maternal care (increased by disturbing pups), temperature changes, and/or by stimulation of stress pathways (reviewed and discussed in Macrì \& Würbel, 2006; Pryce \& Feldon, 2003; Russell, 1971); few have also considered the further possibility of early habituation to humans (Abel, 1971), or "taming".

As part of standard husbandry, cage-cleaning for breeding rats typically involves a human lifting the pups and parent(s) out of their nest and placing them into new bedding, sometimes via a holding area; the mother then collects her pups together and creates a new nest. Thus,

DEV06-107.R2(20279) 
although cage-cleaning usually involves briefer handling than EH treatments, like EH, it involves tactile stimulation, cooling, a change in maternal behavior, human contact causing no lasting harm, and potentially, stimulation of the stress pathways (e.g., Burn, 2006; Gamble, 1982; Van Loo et al., 2000). Perhaps because of this, control rats in EH studies typically receive no handling, including no cage-cleaning, before weaning (Pryce \& Feldon, 2003). Indeed rat pups experiencing "normal husbandry" or "animal facility rearing" (including cagecleaning) show the reduced anxiety expected in EH rats on most tests (e.g., Plotsky et al., 2005), although they also show increased fear-conditioned freezing (reviewed in Pryce \& Feldon, 2003) and sometimes an increased corticosterone stress response compared with EH rats (Lehmann et al., 2002).

These findings raise the possibility that differing early experiences stemming from normal husbandry could have lasting effects on adult phenotypes, potentially contributing unexplained variance in research results. Under nonexperimental conditions, cage-cleaning frequencies in different institutions can vary between three times per week (or very rarely daily) and once every two weeks (especially in individually ventilated cages) (Burn, Day, Peters, \& Mason, 2006a; Burn, Peters, \& Mason, 2006b). This would affect the absolute number of cage-cleaning (and associated handling) episodes experienced before weaning, and also the age at which this first occurs. Perhaps then, more frequent early cage-cleaning induces lasting reductions in rat anxiety. Indeed, Rex, Sondern, Voigt, Franck, and Fink (1996) found that the anxiety levels of identical strains of rats differ depending on which supplier they are obtained from. However, such husbandry practices are typically not reported in research publications.

Currently, there is little information on whether the number of EH episodes influences the magnitude of anxiety reduction in adults. It is also not yet clear whether the age at first handling is important: Meaney and Aitken (1985) found that handling on postnatal Days 1-7 increased hippocampal glucocorticoid receptor levels as much as handling for the full 20 days, while handling on Days 15-21 had no effect. Conversely, corticosterone responses to stress were reduced by handling, whether before or after weaning (Ader, 1970). Our aim was therefore to explore whether variation in these aspects of early experience, representing typical practice in commercial breeding establishments, has lasting affects on adult rats' anxiety profiles. We used four anxiety/stress tests. The first two, which reliably differentiate $\mathrm{EH}$ and non-handled $(\mathrm{NH})$ rats, were the elevated plus-maze (reviewed in Fernandez-Teruel et al., 2002; Pryce \& Feldon, 2003) and hyponeophagia test (Bodnoff, Suranyi Cadotte, Quirion, \& Meaney, 1987; Caldji, Francis,
Sharma, Plotsky, \& Meaney, 2000; Ferré et al., 1995; Macri, Mason, \& Wurbel, 2004; McIntosh, Anisman, \& Merali, 1999; Weinberg, Smotherman, \& Levine, 1978, but see Hilakivi Clarke, Turkka, Lister, \& Linnoila, 1991). The third measure was baseline corticosterone concentration, which can be reduced by EH (Plotsky \& Meaney, 1993, but see Macri et al., 2004). Finally, we used a human interaction test because EH rats struggle, squeak, and defecate less during handling than NH ones (Eells, 1961; Ferré et al., 1995). We measured handleability, but also a novel physiological response to handling: the Harderian gland secretion, chromodacryorrhoea ("red/bloody tears") (e.g., Clement, 1994; Harkness \& Ridgway, 1980; Mason, Wilson, Hampton, \& Wurbel, 2004). Chromodacryorrhoea has only recently started to be used explicitly as a measure of negative affect (Mason et al., 2004), increasing in response to a diverse set of negative events (reviewed in Burn, 2006) and being closely linked to the parasympathetic stress system (e.g., Clement, 1994; Harkness \& Ridgway, 1980; Santos \& Carlini, 1988).

Finally, since there was some prior evidence that individual identification schemes can affect animal physiology and behavior (e.g., Lacey, Beynon, \& Hurst, 2007; Moorhouse \& Macdonald, 2005; Murray \& Fuller, 2000), we chose to use a marking scheme that left one rat unmarked per cage. This enabled any marking effect to be later controlled for statistically.

\section{EXPERIMENT 1}

\section{Methods}

Animals. The Wistar rats in this study were born in one of two buildings within the establishment of a commercial supplier (Harlan, Bicester, UK) as described in (Burn \& Mason, in press). Their parents were pair-mated and kept in polypropylene cages $\left(51 \times 32 \times 20 \mathrm{~cm}^{3}\right)$ with stainless steel wire lids, and autoclaved Lignocel 34 sawdust (J. Rettenmaier \& Söhne, Holzmühle, Germany). Pelleted chow (2018S sterilizable, Harlan Teklad, Bicester, UK) and water were provided ad libitum. Their cages were cleaned on one of three schedules: either twice weekly, once weekly, or every 2 weeks (fortnightly). There were 18 cages of each treatment in each building, giving a total of 108 cages. Cage cleaning involved replacing the polycarbonate base with a clean one containing fresh sawdust, but the lids were retained. The handling initially involved the technician scooping up neonates as a group in their nest, and from when the pups were about 4 days old they were picked up individually by the body. The supplier provided us with information on the precise number of cage-cleanings each rat had received before weaning, and also the age at which their first cagecleaning occurred. Rats were weaned at 3-4 weeks of age 
in weekly batches because the births were staggered over time, and the size of each batch varied depending on how many litters were ready for weaning each week.

One male pup from the third litter of each pair was transported to an animal unit at the University of Oxford (males were used because estrus can suppress the EH effect in females (Severino et al., 2004)). We obtained a total of 92 unrelated rats, delivered in 8 batches over the course of 6 weeks. Once in the animal unit, the rats were housed in same-treatment pairs (or on four occasions triple-housed, when a batch consisted of an odd number). Cages were polypropylene $\left(50 \times 32 \times 25 \mathrm{~cm}^{3}\right)$ with stainless steel wire lids, and aspen woodchip bedding $8 / 20$ (Lillico, Surrey, UK). Pelleted chow (PCP mod (P), Special Diet Services, Witham, UK) and water were provided ad libitum. Nesting material (Sizzle Nest, Datesand, Manchester, UK) and cardboard tubes (Maxi Fun Tunnel, Lillico) were provided as environmental enrichment. The room was maintained at $21 \pm 1^{\circ} \mathrm{C}$, with a humidity of $50 \pm 10 \%$, and the light-dark cycle was 12:12 hr, with the lights on at $7 \mathrm{am}$.

One rat (or two of the triple-housed rats) per cage was tail-marked for individual identification using a black permanent marker (Pentel N50, Pentel Co. Ltd., Swindon, UK). This is considered a fairly standard, "low-tech" method for marking rodents (Wang, 2005). The marking procedure consisted of a seated technician lightly restraining each rat between his arm and body, with the nose of the rat towards his elbow. The mid-section of the tail was supported in one hand while the other applied the black marker pen in caudally directed strokes to make a mark approximately $1 \mathrm{~cm}$ long. The marking scheme was random with respect to rat behavior, because the rats were delivered in individual boxes, the cages were filled sequentially, and the second rat of each pair was marked as it was allocated to its cage upon arrival at our laboratory. Marks were replenished every $2-3$ weeks as required.

All cages were cleaned weekly, and the experimenter and technicians were blind as to which rats belonged to which treatment group. For all the tests, the order in which individuals were tested was pseudo-randomized according to treatment groups and other factors, although cagemates were tested consecutively. All testing was conducted in the holding room to minimize any effects of transport stress, and the cages were removed from their holding racks $5 \mathrm{~min}$ before testing to ensure that the rats were alert.

Human Interaction Test. At 12-14 weeks of age, the rats' regular technician brought the rats to the front of their cage by gently tapping a pen on the cage bars, to inspect their baseline nasal chromodacryorrhoea. The amount of chromodacryorrhoea was scored using an extended adaptation of the system validated by Mason et al. (2004), as follows. A photographic guide was also provided to illustrate the scoring system (in Burn, 2006).

0 No visible chromodacryorrhoea

1 One small ( $<1 \mathrm{~mm}$ in diameter) droplet of chromodacryorrhoea

2 One larger droplet or $\leq 4$ small droplets of chromodacryorrhoea

$3 \geq 2$ large droplets or $\geq 5$ small droplets of chromodacryorrhoea

$425-50 \%$ of the nose covered by chromodacryorrhoea

5 Over $50 \%$ of nose covered in chromodacryorrhoea.

Each rat was then picked up and allowed to explore the technician's forearm for $30 \mathrm{~s}$, being gently restrained by the base of the tail. The technician scored the ease of handling, using a handleability scoring system (developed in collaboration with experienced technicians) as described below.

1 Very relaxed: no struggling or squeaking, but alert

2 Easy to handle: little struggling or squeaking

3 Reactive: tense with some struggling and squeaking

4 Resistant to handling: struggled and squeaked

5 Very resistant to handling: struggled, attempted to bite, squeaked.

After 10 min (an appropriate interval as indicated by our pilot studies (Burn, 2006), and before most rats would start to groom after handling (Burn et al., 2006b)), the technician returned to each cage, bringing the rats to the front as before, and rescored their chromodacryorrhoea.

Elevated Plus-Maze Test. On the following day, the elevated plus-maze test was carried out. The maze was wooden, painted with waterproof gray paint, and left to dry for 3 weeks before the start of the experiments to prevent any solvent vapors affecting the rats' behavior (Heale, Vanderwolf, \& Kavaliers, 1994; Zibrowski \& Vanderwolf, 1997). The maze was elevated by $50 \mathrm{~cm}$, and each arm was $50 \mathrm{~cm}$ long and $10 \mathrm{~cm}$ wide, with $45 \mathrm{~cm}$ walls on the closed arms, and $5 \mathrm{~cm}$ rims on the open arms (dimensions the same as those in most of the EH literature). Before testing, five nonexperimental male rats were placed onto the maze for $5 \mathrm{~min}$ to minimize the difference in overall rat odor intensity between the first and last of the test rats. The experimental rats were placed into a closed arm of the maze, facing the end wall. The observer (the rats' regular technician) sat quietly about 1 $m$ away from the maze, between an open and a closed arm. We took only those measures that we felt were least ambiguous to interpret (Wall, 2001) to minimize the risk of falsely significant results (Type I errors). The observer 
thus recorded latency to emerge from the starting arm, latency to enter the first open arm, proportion of time spent in the open arms, defecation, and urination. An arm entry was defined as the rat placing all four feet into the new area. The central square was not recorded as a separate area due to its ambiguity in relation to anxiety (Wall, 2001) (e.g., if a rat moved onto the central square but never moved onto an open arm, it was scored as spending all its time in the closed arms). The one exception to this was the latency to emerge from the original closed arm, which was recorded to allow us to discriminate for example between any rats that remained virtually immobile throughout the test, and those that explored both closed arms. The test lasted for $5 \mathrm{~min}$, after which the fecal pellets and urine were removed. The apparatus was then cleaned with a damp tissue and a dry one between rats.

Baseline Corticosterone. We measured fecal (rather than serum or urinary) corticosterone to avoid invasive handling procedures. Fecal pellets were collected from the human interaction test and the elevated plus-maze for baseline corticosterone analysis. This would give us a baseline measure, since although there is wide variance in the delay between serum and fecal corticosterone concentrations, serum levels are not reflected in the fecal boli until $16.7 \pm 4.3 \mathrm{hr}$ after a stressor (Bamberg, Palme, \& Meingassner, 2001). The pellets were immediately placed into sealed $50 \mathrm{ml}$ polypropylene tubes, and within a maximum of $2 \mathrm{hr}$, frozen at $-20^{\circ} \mathrm{C}$ for up to 8 weeks before being sent for professional corticosterone assaying (Gordon Laboratory Group, Stockton-on-Tees, UK).

Hyponeophagia Test. On the third day of testing, a hyponeophagia test similar to previous studies (Caldji et al., 1998, 2000; Francis, Diorio, Plotsky, \& Meaney, 2002; Rex, Voigt, Voits, \& Fink, 1998) was carried out. Canned sweetcorn (Green Giant Original, with added sugar and salt) was the novel food. However, from the first batch of rats it became clear that rats in this study were much more neophobic than those in previous studies (only 2 out of the 13 rats ate the corn). Therefore, due to a ceiling effect in the latencies, the first batch of rats was excluded from analyses. Subsequent batches of rats were fooddeprived overnight (for 14-18 hr) and the test was adjusted so as to reduce the degree of novelty involved. Using a design similar to that described by McIntosh et al. (1999), each rat's cagemate was removed to a holding cage, and five sweetcorn kernels were presented in a brown, ceramic dish, $8 \mathrm{~cm}$ in diameter, at the back of the homecage. The food and its container were now the novel stimuli, but the environment was familiar. The technician recorded the latency to start unambiguously eating (holding a kernel in the forepaws and eating for at least $2 \mathrm{~s}$ ). Test duration was a maximum of $5 \mathrm{~min}$.
When the second (or third) cagemate was tested, it was prevented from contacting the first cagemate before participating in the test, to avoid social facilitation through smelling the novel food on its cagemate's breath (Galef, Mason, Preti, \& Bean, 1988). Between tests the bowl was cleaned with a damp tissue and a dry one, and any remaining sweetcorn was replaced with fresh kernels, again to avoid the influences of any odor deposits from previous rats.

Statistical Methods. The statistical program used was Minitab $^{\text {TM }}$ version 14 (Minitab Ltd., Pennsylvania, USA). Latencies and durations were arcsine and $\log$ transformed, respectively, to normalize the residuals. General linear models were used to test the fixed effects of the number of cage-cleanings before weaning, whether or not rats were marked, and which building they were born in. Interactions between number of cage-cleanings, marking, and building were also included. Number of cage-cleanings was treated as a covariate, after graphically inspecting the residuals in the case of nonlinear relationships. Batch and building were controlled for in statistical analyses because such composite factors can affect test results (e.g., Burn et al., 2006a; Chesler, Wilson, Lariviere, Rodriguez Zas, \& Mogil, 2002; Crabbe, Wahlsten, \& Dudek, 1999; Rex et al., 1996). Batch was nested in building because earlier batches came from Building A and later ones from B. Cage, a random factor nested in Batch, was included to control for nonindependence of cage-mates. These tests were then repeated with "age at first cage-cleaning" instead of the number of cage-cleanings, and the models were compared; the two variables could not be used together because they were so tightly confounded with each other (i.e., more frequent cleaning was associated with earlier cleaning events).

To test for pairwise relationships between measures from the different tests, the full GLMs were rerun, but this time each test result was included in turn. For example, to test the relationship between time spent on the plus-maze open arms and latency to feed in the hyponeophagia test, one was used as the response, and the other was used as a predictor alongside the background of all the blocking factors.

Where the most relevant literature provided equivalent effect sizes, important nonsignificant findings were tested against those hypothesized effect sizes using power tests for two-sample tests (comparing the most and least frequent cage-cleaning). Using the software available to us, power tests for more controlled experimental designs or those using covariates were impossible, so these tests are conservative, that is, we should have been able to detect more subtle effects than they suggest. Posthoc power tests to compare findings against a hypothesized or 
biologically significant effect size provide conflicting evidence to $p$-values (e.g., Hoenig \& Heisey, 2001), so here we use them merely as an additional way of expressing our findings. We also provide confidence intervals for important nonsignificant findings (Colegrave \& Ruxton, 2003).

\section{RESULTS}

\section{Correlations Between Test Measures}

Within Tests. In the human interaction test, the increase in chromodacryorrhoea was greater in rats that were more difficult to handle $\left(F_{1,27}=5.71 ; p=.024\right)$ (Fig. 1). In the plus-maze, latency to emerge from the initial closed arm correlated positively with latency to enter an open $\operatorname{arm}\left(F_{1,44}=9.18 ; p=.004\right)$, and in Marked rats only, the latency to enter an open arm correlated negatively with time spent in the open arms $\left(F_{1,43}=4.88 ; p=.033\right)$. In the hyponeophagia test, feeding latency correlated negatively with whether or not rats ate $\left(F_{1,30}=36.73 ; p<.001\right)$.

Between Tests. Rats that spent more time in the open arms of the plus-maze were more likely to eat sweetcorn in the hyponeophagia test $\left(F_{1,26}=5.00 ; p=.034\right)$ and had shorter latencies to do so $\left(F_{1,26}=6.56 ; p=.017\right)$. No other intertest relationships were found.

\section{Human Interaction Test}

There were no significant effects of any independent variable including cleaning frequency and age at first

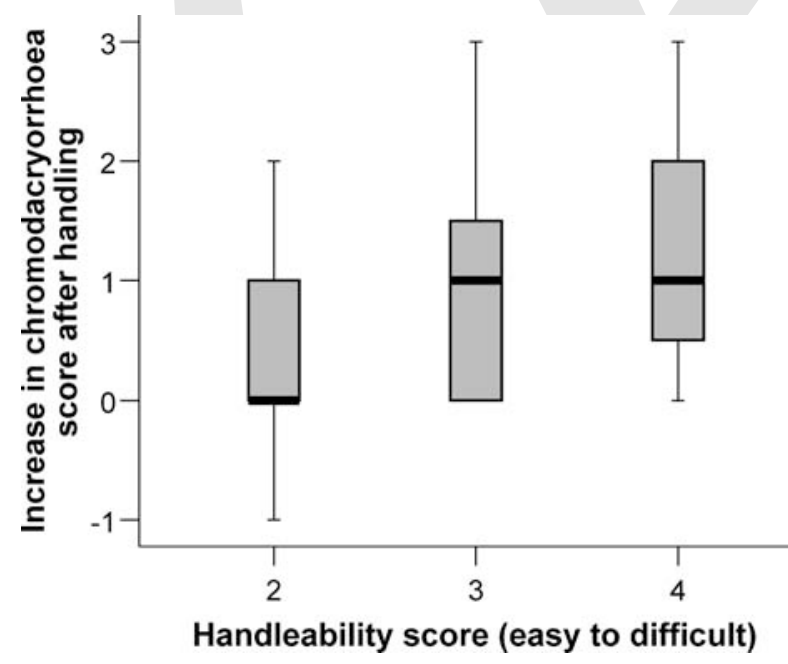

FIGURE 1 The relationship between handleability and the increase in chromodacryorrhoea score (median \pm interquartile range) in the human interaction test. Rats that were easier to handle (scoring 2) had less pronounced chromodacryorrhoea responses to handling than those that were more difficult to handle (scoring 4). cage-cleaning on handleability scores (e.g., number of cage-cleanings: $F_{1}, 42=.05 ; p=.818 ; 95 \% \mathrm{CI}$ for the slope $=-.15, .19)$. Most rats scored 2 or 3 (36 and 44 , respectively), and none scored 5: "very resistant".

Rats had higher chromodacryorrhoea scores $10 \mathrm{~min}$ after handling than before $\left(F_{1}, 62=48.48 ; p<.001\right)$. Marked rats had a larger increase in chromodacryorrhoea following handling than the unmarked ones $\left(F_{1,28}=\right.$ 13.26 ; $p=.001$ ) (Fig. 2), but again there were no cagecleaning effects $\left(F_{1,28}=2.39 ; p=.133 ; 95 \% \mathrm{CI}\right.$ for the slope $=-.023, .49)$.

\section{Elevated Plus-Maze Test}

Cage-cleaning frequency and age at first cleaning had no effects on any plus-maze behavior (e.g., effects of number of cage-cleanings on time spent on open arms: $F_{1}$, $44<.01 ; p=.99 ; 95 \% \mathrm{CI}$ for the slope $=-.05, .034)$ (see Fig. 3A for the lack of a positive trend). There were also no significant effects of any variables on urination or defecation. Marked rats, however, had lower latencies to emerge from the closed arm they were initially placed into $\left(F_{1,41}=17.96 ; p<.001\right)$, lower latencies to enter the open arms $\left(F_{1,42}=10.69 ; p=.002\right)$, and spent a significantly higher proportion of time in the open arms $\left(F_{1,42}=23.19 ; p<.001\right)$ than unmarked rats (Fig. 4). For example, $20.9 \pm 2.9 \%$ of the marked rats' time was spent on the open arms, compared with only $7.5 \pm 1.4 \%$ of the unmarked rats' time (Fig. 4C). Batch also affected the proportion of time spent in the open arms $\left(F_{6,39}=5.14\right.$; $<.001)$ and the latency to enter an open $\operatorname{arm}\left(F_{6,39}=2.94\right.$; $p=.015)$, although there was no obvious temporal trend spanning between earlier and later batches.

Power tests showed that our study would have been able to detect an effect size comparable to that found by

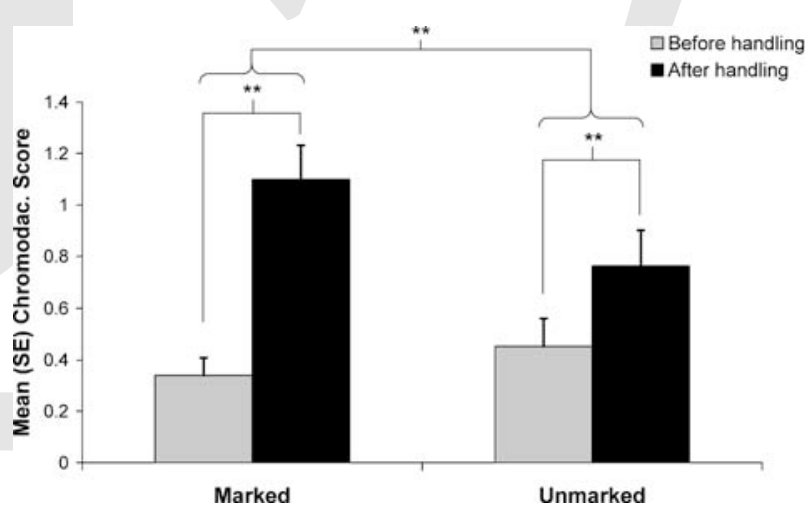

FIGURE 2 Nasal chromodacryorrhoea scores (mean $\pm \mathrm{SE}$ ) before and $10 \mathrm{~min}$ after handling in rats that were marked or unmarked for individual identification purposes. Chromodacryorrhoea increased after handling in both groups, but this increase was more pronounced in marked rats than unmarked ones. $^{* *}$ indicates that $p \leq .001$. 
A

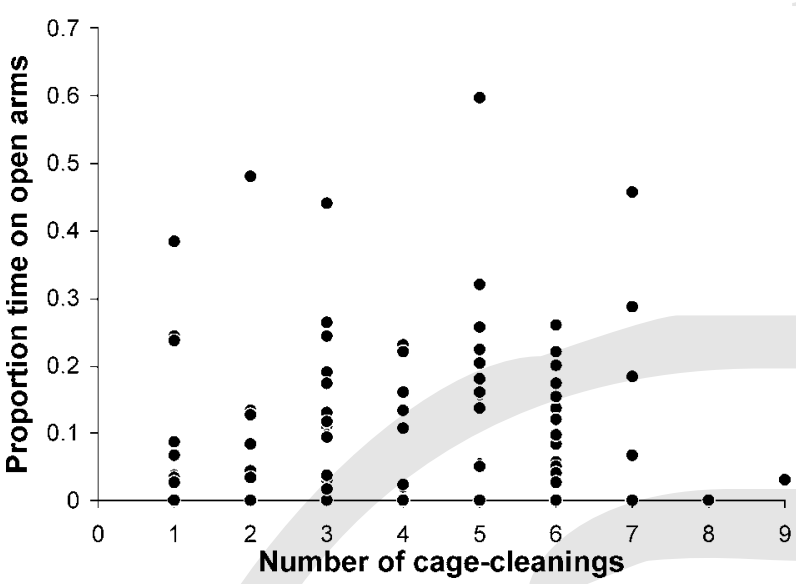

B
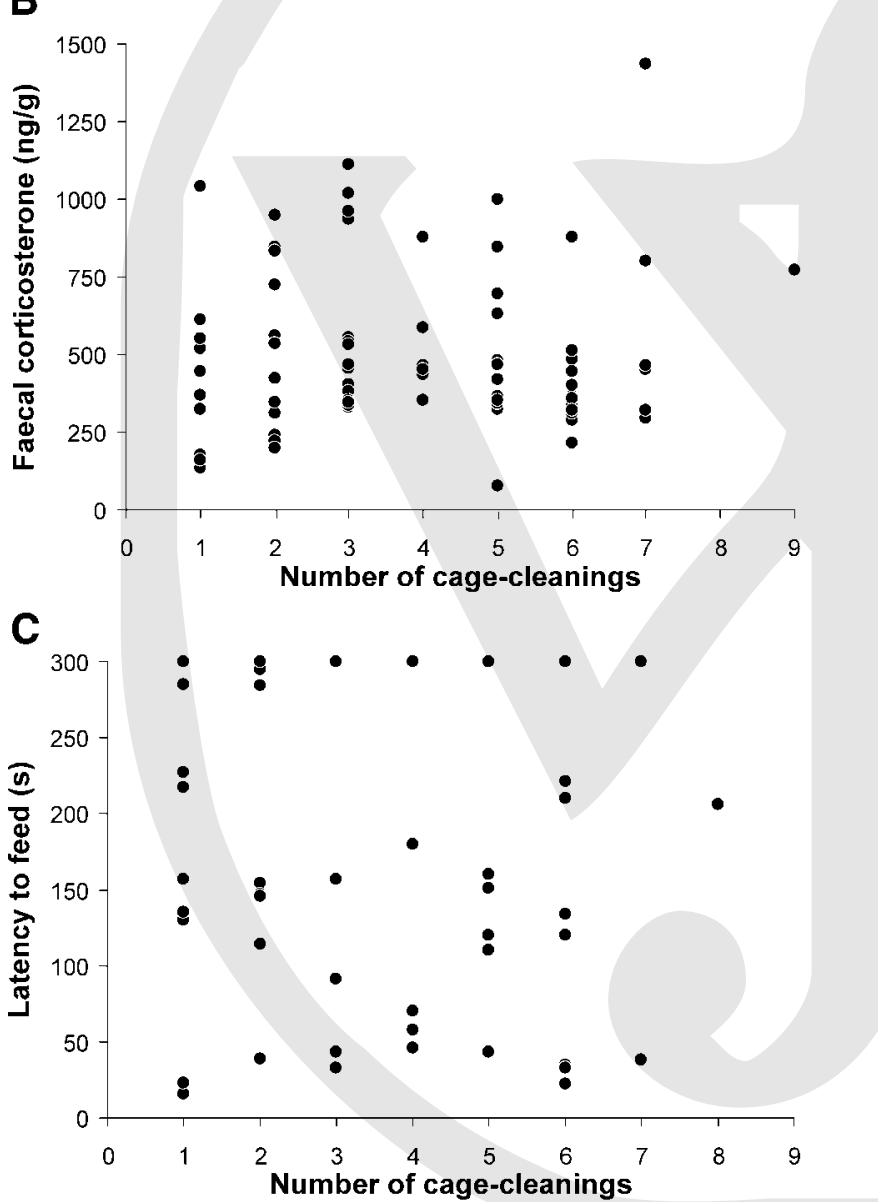

FIGURE 3 Example scatter plots showing the (lack of) trends between the number of early cage-cleanings and adult rat anxiety measures: (A) the proportion of time spent on the open arms of the elevated plus-maze, $(\mathbf{B})$ the baseline fecal concentration of corticosterone, and $(\mathbf{C})$ the latency to feed in the hyponeophagia test.
A

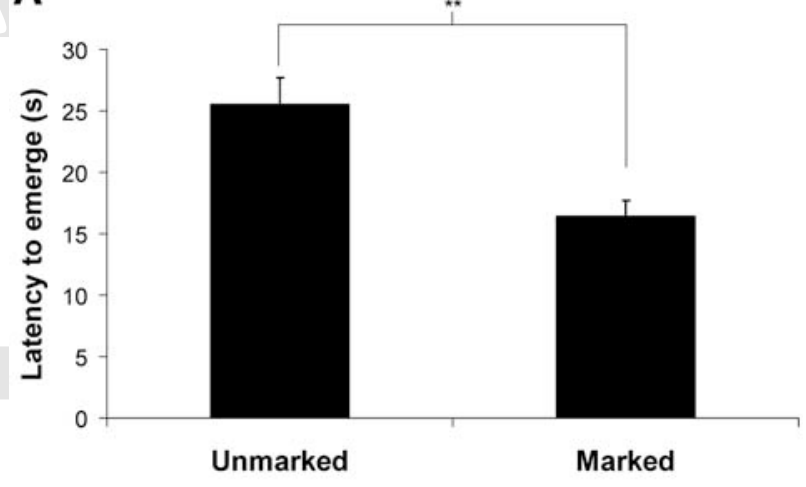

B
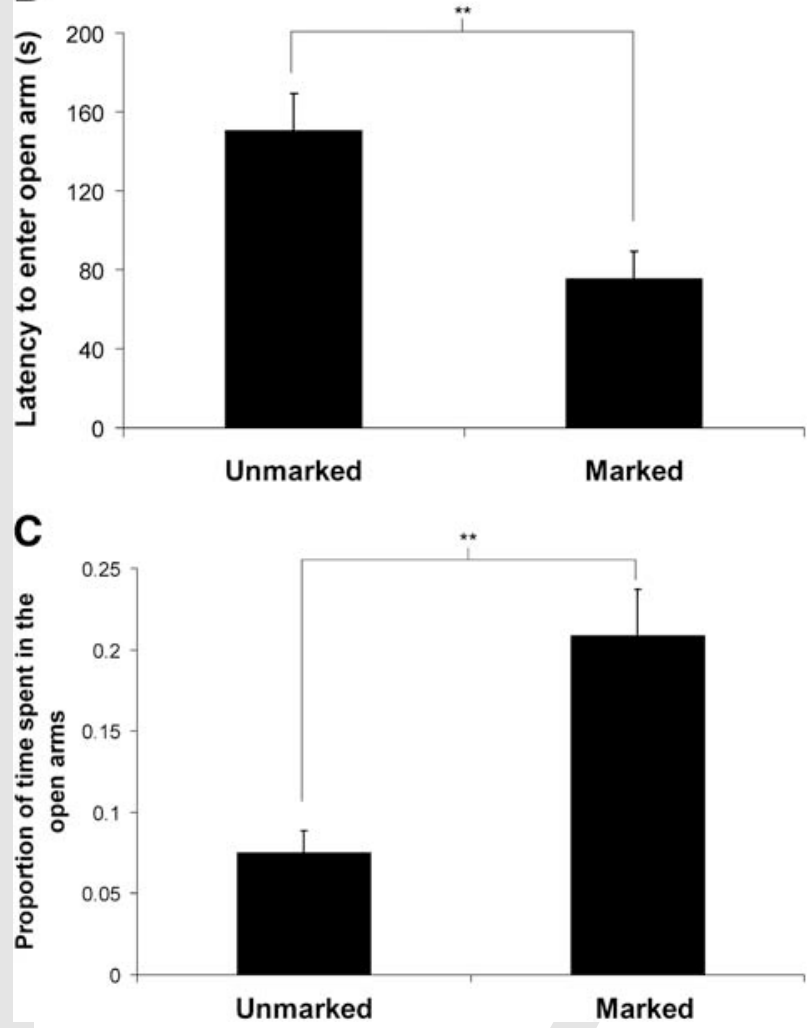

FIGURE 4 The plus-maze behavior of rats that were individually marked versus those that were not marked. Compared with unmarked rats, marked rats had significantly (A) lower latencies to emerge from the closed arm they were initially placed into, (B) lower latencies to enter the open arms, and $(\mathbf{C})$ they spent a significantly higher proportion of time in the open arms. All results are presented as a mean $\pm \mathrm{SE}$; ${ }^{* *}$ indicates that $p \leq .001$. 
McIntosh et al. (1999) (who were kindly able to provide accurate means for their data), as an example of a similar test set-up. Thus, even using only our unmarked rats (similar to McIntosh et al., 1999), we would have had a power of over .98 to detect the magnitude of difference they report in time spent on the open arms of the plus maze between $\mathrm{EH}$ and $\mathrm{NH}$ rats $(n=14$ per cleaning treatment group (unmarked only); hypothesized effect size $=31.5 \mathrm{~s}$; standard deviation $=20.1 ; \alpha=.05$ ). This indicates that our nonsignificant result was unlikely to have been a Type II error.

\section{Baseline Corticosterone}

There were no effects of any variables on fecal corticosterone levels (e.g., number of cage-cleanings: $F_{1,35}=$ $.05 ; p=.831 ; 95 \% \mathrm{CI}$ for the slope $=-1.53,1.98$ ) (Fig. 3B illustrates the lack of any negative trend).

\section{Hyponeophagia Test}

Only 40 of the 64 rats that experienced the final test design $(62.5 \%)$ ate sweetcorn in this test, and whether or not rats ate the sweetcorn was not affected by any variable. Similarly, of those that ate the corn, there were no significant effects of any variable on their latencies to eat (e.g., number of cage-cleanings: $F_{1,28}=.64 ; p=.431$; $95 \% \mathrm{CI}$ for the slope $=-.27, .10)($ Fig. $3 \mathrm{C}$ shows the lack of a negative trend).

Power tests showed that our study would have been able to detect an effect size comparable to that reported by Caldji et al. (2000). Including marked and unmarked rats (because marking had no significant effect on this test), we would have had a power of .99 to detect the magnitude of difference they report in latency to feed between EH and $\mathrm{NH}$ rats (minimum $n=19$ per cleaning treatment group; hypothesized effect size (arcsine transformed) $=.73$; standard deviation $=.49 ; \quad \alpha=.05$ ). Using only our unmarked rats (minimum $n=9$ ), we would still have had a power of .87. This is despite the large number of missing values for this test.

\section{EXPERIMENT 2}

To further investigate the effect of tail-marking, an experiment was devised to measure the aversiveness of marker pens. It is known that a variety of organic solvents are aversive to rodents (Heale et al., 1994; Zibrowski \& Vanderwolf, 1997). Therefore, Experiment 2 was devised to investigate the aversiveness of the marker pen odor. This experiment was performed a year after Experiment 1. Although these were a different strain to the Wistars used in Experiment 1, the question being asked was whether the odor of marker pens is aversive to rats generally, rather than a more specific strain-related question. These hooded rats were obtained from the same supplier as the Wistars (Harlan, UK). Moreover, the hooded rats had the advantage that they could be identified by their distinctive coat markings, since it was clearly undesirable to tailmark them with a marker pen for identification purposes.

\section{Methods}

Animals. Ten male hooded Lister outbred rats (Harlan, UK), weighing 427-605 $\mathrm{g}$ at the time of testing, were used. They were housed in groups of 2-3 in the conditions described in Experiment 1. They were identified by noting their distinctive coat markings.

Aversion Test. A traditional two-choice Grice box arrangement was used. It was made from aluminum sheet and was $20 \mathrm{~cm}$ high. A start box, $30 \times 14 \mathrm{~cm}^{2}$, led into a choice area which widened over a length of $8 \mathrm{~cm}$ from $14 \mathrm{~cm}$ width at the start box end to $27 \mathrm{~cm}$ width where it led to two adjacent goal boxes, each $30 \times 13.5 \mathrm{~cm}^{2}$. Each goal box contained four Pentel N50 permanent marker pens, as in Experiment 1, bundled together. These pens had not previously been used to mark rats so they would not have been contaminated with rat odor. In the "Control" box the pens were capped, and in the "Odor" box, they were uncapped to allow odor/solvent release. The pens were uncapped at least $1 \mathrm{~min}$ before the first rat was placed in the apparatus, to allow the odor to diffuse into the surroundings.

After weighing, the rats were taken from the stock room to a nearby holding room outside the test room, where they were left for approximately $10 \mathrm{~min}$ to become fully alert and acclimatized to the new surroundings. They were then individually placed into the start box for a $2 \mathrm{~min}$ trial, in which free exploration of the apparatus was permitted. The latency to enter each goal box (all four feet) and the time spent there (end point: all four feet out of goal box) were recorded, as was the number of entries into each box. Defecation and urination were also recorded.

Statistical Analysis. General linear models were used to compare each behavior between the Odor and Control boxes, controlling for the side (left or right) on which the uncapped pens were presented, and the cage the rats came from. Individual rats were included as random factors nested within cage and the side of presentation. The model assumptions were checked graphically, and latency data were $\log$ transformed as required.

\section{RESULTS}

Results are presented in Table 1. Rats had significantly longer latencies to visit the Odor box, spent less time in it, and visited it less frequently than the Control box 
Table 1. Results of the Grice Box Test of Rats' Avoidance of Marker Pen Odor

\begin{tabular}{lccc}
\hline Measure & Mean $\pm \mathrm{SE}$ & $F$-value (DF, Error) & $p$-value \\
\hline Latency Control (s) & $22.5 \pm 3.8$ & & .004 \\
Latency Odor (s) & $74.3 \pm 12.5$ & $14.02(1,9)$ & .001 \\
Time in Control (s) & $49.7 \pm 5.4$ & $24.06(1,9)$ & .001 \\
Time in Odor (s) & $13.5 \pm 3.3$ & & \\
Entries into Control & $2.1 \pm .2$ & $22.50(1,9)$ &. \\
Entries into Odor & $1.1 \pm .2$ & & \\
\hline
\end{tabular}

Note. The significance level was set at $p=.05$.

(Table 1). Behavior was similar in most rats; 9 of the 10 rats entered the Control box first, explored it, and then returned to the choice area. From here, they would generally enter the Odor box, but one of the rats never entered this box and another only entered it for $1 \mathrm{~s}$. Only one rat defecated in the apparatus, and one other urinated.

\section{DISCUSSION}

In this study, we investigated whether the cage-cleaning frequencies of rat pups would affect their anxiety profiles in adulthood. Despite some prior evidence that variation in the frequency or timing of EH can induce variation in physiology (see Introduction: Denenberg \& Karas, 1959; Meaney \& Aitken, 1985), and despite testing over 90 rats that experienced cage-cleaning between one and nine times preweaning, we found no such effects. Failing to find an effect does not, of course, prove that no effect exists. Type II errors might be most likely in our baseline fecal corticosterone concentration, which had a noticeably wider confidence interval that the other measures: most differences in $\mathrm{EH}$ are found with stress-induced corticosterone responses, so basal differences are relatively subtle if they are observed at all (e.g., Macri et al., 2004; Plotsky \& Meaney, 1993). Also, although the relative variation in fecal corticosterone concentrations is comparable to variation in plasma concentrations, it includes a longer timescale, with accompanying variation in rats' experience and circadian rhythm (Bamberg et al., 2001; Cavigelli et al., 2005).

However, our power tests suggest that at least for plus maze and hyponeophagia data, we had more than a $98 \%$ chance of detecting an effect of the magnitude found between EH and NH rats (c.f. McIntosh et al., 1999; Caldji et al., 2000). Our lack of differences between cleaning treatments was therefore probably not an artifact of low power, especially since EH reductions in stress/anxiety have been found using groups of as few as 10 (McIntosh et al., 1999) or 8-10 (Kalinichev, Easterling, Plotsky, \& Holtzman, 2002) rats in the elevated plus-maze, and 8 (McIntosh et al., 1999) or 7-10 (Caldji et al., 2000) rats in the hyponeophagia test. In fact, in the latter study, Caldji and colleagues found that NH rats had approximately $70 \%$ longer latencies to feed than $\mathrm{EH}$ ones, and yet the cagecleaning frequencies used here have shown no discernable trend in the same direction (Fig. 3C). While some effects might have emerged had we taken other measures, such as corticosterone responses to stress (e.g., Huot, Thrivikraman, Meaney, \& Plotsky, 2001; Kalinichev et al., 2002; Macri et al., 2004; Vallee et al., 1997), overall we consider this unlikely given the lack of effects we found across four behavioral and physiological responses usually affected by $\mathrm{EH}$. Thus, we conclude that the variation in handling and cage-cleaning likely to occur between rodent breeding facilities does not seem to have lasting affects on male rats' anxiety profiles.

This apparently contrasts with previous findings comparing NH controls and animals experiencing standard husbandry; rat pups undergoing weekly or twice weekly cage-cleaning have reduced anxiety levels compared with $\mathrm{NH}$ animals, similar to EH rats, at least in most respects (reviewed in the Section Introduction). One possibility is that experiencing cage-cleaning eight or nine times before weaning might be no more anxiolytic than experiencing it only once or twice. However, another possible explanation is that the current "real life," applied study involved additional factors that do not usually occur in controlled, experimental EH studies, and these might mask any subtle differences between the cage-cleaning frequencies. One factor could be the postweaning transport (a journey of $25 \mathrm{~km}$ by road) between the breeding company and our laboratory. In rodents, transport can cause prolonged physiological and behavioral stress responses (DeSantis \& Schmaltz, 1984; Hayssen, 1998; Landi, Kreider, Lang, $\&$ Bullock, 1982), and therefore may have been powerful enough to obscure any subtle effects of previous experience on later anxiety levels. Future research could investigate whether early cage-cleaning frequency affects the later anxiety profiles of rats tested without transportation to a new site. However, overall, since cagecleaning frequencies in our realistic set-up did not have differential effects on rat anxiety levels, we can conclude that it is unlikely to be the mechanism by which samestrain rats bought from different commercial suppliers show different anxiety profiles (Rex et al., 1996). 
We did, however, find two novel effects on rat anxiety. The first was that the delivery batch that the rats arrived in affected the elevated plus-maze measurements. These effects could have originated from a number of sources including the specific weather and traffic conditions when each batch was transported to our laboratory, or genetic variation in this outbred strain; the effects were not linearly related to batch, so the variation was not due to a gradual directional change. It is unclear why delivery batch only affected plus-maze results, but the four tests probably quantify different aspects of anxiety, fearfulness, or negative affect (File, 1992; Gray \& McNaughton, 2000; Ramos, Berton, Mormede, \& Chaouloff, 1997; Võikar, Polus, Vasar, \& Rauvala, 2005).

Our second unexpected finding was that marked rats entered the open arms of the plus-maze more readily than unmarked rats, suggesting lower anxiety, and yet they had higher chromodacryrrhoea responses to handling which paradoxically suggests that some form of negative affect was increased. Marking with permanent marker required renewing three or four times between weaning and testing, so marked rats could have appeared bolder in the plus-maze because they received extra handling. Habituation to handling per se is already known to reduce rats' anxiety in the elevated plus-maze (Andrews \& File, 1993; Brett \& Pratt, 1990; File, Andrews, Wu, Zharkovsky, \& Zangrossi, 1992; Hogg, 1996). Therefore, the marking effect could imply that supplementary handling increases open arm entry, regardless of whether it occurs before weaning, as with $\mathrm{EH}$, or afterwards, as was found by Ader (1970; but see Meaney \& Aitken, 1985). However, this will require confirmation within a controlled study.

The increased chromodacryorrhoea secretion in the marked rats also suggests that some aspect of being marked must have been aversive or stressful in some way. Handling is often stressful for laboratory animals (Balcombe, Barnard, \& Sandusky, 2004), and indeed chromodacryorrhoea increased after handling in both groups of rats. The results of Experiment 2 also demonstrate that rats avoid the odor of at least some types of marker pen ink, including the one used here. If being marked is aversive, it could have caused the marked rats to form stronger negative associations with handling than unmarked rats (accounting for marked rats' heightened chromodacryorrhoea responses), while at the same time perhaps conferring more experience of coping with challenges generally (accounting for their relatively bold behavior in the plus-maze). Aversive experiences could leave indelible "primary" associations, while concurrently providing partial resistance to secondary or unrelated aversive contexts.

On a physiological level, because chromodacryrrhoea secretion occurs through increased parasympathetic activity, (e.g., Clement, 1994; Harkness \& Ridgway, 1980; Santos \& Carlini, 1988), perhaps parasympathetic activity was altered in the marked rats. Further research could examine, physiologically, if this was indeed the case. Further research could also investigate what aspect(s) of marking underlie its effects on both behavioral and physiological responses. For example, the marking process could have caused physical discomfort through manual restraint or slight tail-pinch (e.g., D'Angio, Serrano, Rivy, \& Scatton, 1987), the ink may have contained solvents that were anxiogenic (Heale et al., 1994; Zibrowski \& Vanderwolf, 1997) or slightly toxic (Anderson \& Anderson, 2003), or the marks could have affected cagemates' social relationships (Lacey et al., 2007). Already, the results of Experiment 2 demonstrate that some pens used to mark rats do release an aversive odor.

Both our novel findings concerning marking and batch effects were unexpected, not based on our initial hypotheses, so further research is needed to verify whether they are replicable, and to elucidate the mechanisms underlying them. However, our results do add to accumulating evidence that a variety of marking methods can have profound effects on animals (e.g., Lacey et al., 2007; Moorhouse \& Macdonald, 2005; Murray \& Fuller, 2000), and that standardization is not easy to achieve (e.g., Burn et al., 2006a; Chesler et al., 2002; Crabbe et al., 1999; Rex et al., 1996). If scientific results are to have general validity, perhaps adherence to an arbitrary, highly specific "standard" is not even to be desired. Testing that results will generalize across laboratories, by incorporating variation systematically into experimental design, may be a more desirable approach (Garner, Thogerson, Mench, \& Würbel, 2006; Wurbel, 2000, 2002).

To summarize, despite well-documented anxiolytic effects of EH, different cage-cleaning frequencies before weaning apparently have little effect on adult rats' anxiety levels. In fact, given that normal husbandry has previously been shown to reduce anxiety almost as much as EH (e.g., Plotsky et al., 2005), our findings suggest that the cagecleaning effect might reach asymptotic levels after only one or two early cleaning experiences. Seemingly then, different cage-cleaning frequencies cannot explain the differing anxiety profiles observed between same-strain rats from alternative suppliers (Rex et al., 1996). In contrast, identification tail-marking of rats may affect their anxiety profiles in a complex and paradoxical manner, and the odor released from marker pens can be aversive to rats. Marking schemes should therefore be considered a potential confound in studies of rodent anxiety. Further work will be required to confirm its effects on rat anxiety, to compare the effects of alternative marking schemes, and to investigate whether it has effects on other aspects of physiology and behavior. 


\section{NOTES}

The Sales and Production Managers at Harlan, UK, and the laboratory managers and animal technicians there and at the University of Oxford are gratefully acknowledged. This work was funded by the UK Home Office. GM was funded by the BBSRC (UK) and then NSERC (Canada). P. Kent, M. Kalinichev, M. Meaney, and Z. Merali helpfully corresponded over their effect sizes to answer our power testing queries. Thanks also to the three anonymous referees for their constructive comments.

\section{REFERENCES}

Abel, E. L. (1971). Habituation as a factor in early handling. Journal of Comparative and Physiological Psychology, 74, 219-221.

Ader, R. (1970). The effects of early experience on the adrenocortical response to different magnitudes of stimulation. Physiology and Behavior, 5, 837-839.

Anderson, R. C. \& Anderson, J. H. (2003). Acute toxicity of marking pen emissions. Journal of Toxicology and Environmental Health Part A, 66, 829-845.

Andrews, N. \& File, S. E. (1993). Handling history of rats modifies behavioural effects of drugs in the elevated plusmaze test of anxiety. European Journal of Pharmacology, 235, 109-112.

Balcombe, J. P., Barnard, N. D. \& Sandusky, C. (2004). Laboratory routines cause animal stress. Contemporary Topics in Laboratory Animal Science, 43, 42-51.

Bamberg, E., Palme, R. \& Meingassner, J. G. (2001). Excretion of corticosteroid metabolites in urine and faeces of rats. Laboratory Animals, 35, 307-314.

Bodnoff, S. R., Suranyi Cadotte, B., Quirion, R. \& Meaney, M. J. (1987). Postnatal handling reduces novelty-induced fear and increases $[3 \mathrm{H}]$ flunitrazepam binding in rat brain. European Journal of Pharmacology, 144, 105-107.

Brett, R. R. \& Pratt, J. A. (1990). Chronic handling modifies the anxiolytic effect of diazepam in the elevated plus-maze. European Journal of Pharmacology, 178, 135-138.

Burn, C. C. (2006). Effects of husbandry manipulations and the laboratory environment on rat health and welfare. Unpublished D.Phil. Thesis, University of Oxford, Oxford, UK.

Burn, C. C., Day, M. J., Peters, A. \& Mason, G. J. (2006a). Long-term effects of cage-cleaning frequency and bedding type on laboratory rat health, welfare, and handleability: A cross-laboratory study. Laboratory Animals, 40, 353-370.

Burn, C. C., \& Mason, G. J. Effects of cage-cleaning frequencies on rat reproductive performance, infanticide, and welfare, (in press).

Burn, C. C., Peters, A. \& Mason, G. J. (2006b). Acute effects of cage-cleaning at different frequencies on laboratory rat behaviour and welfare. Animal Welfare, 15, 161-172.

Caldji, C., Francis, D., Sharma, S., Plotsky, P. M. \& Meaney, M. J. (2000). The effects of early rearing environment on the development of GABA-sub(A) and central benzodiazepine receptor levels and novelty-induced fearfulness in the rat. Neuropsychopharmacology, 22, 219-229.

Caldji, C., Tannenbaum, B., Sharma, S., Francis, D., Plotsky, P. M. \& Meaney, M. J. (1998). Maternal care during infancy regulates the development of neural systems mediating the expression of fearfulness in the rat. Proceedings of the National Academy of Sciences, 95, 53355340 .

Cavigelli, S. A., Monfort, S. L., Whitney, T. K., Mechref, Y. S., Novotny, M. \& McClintock, M. K. (2005). Frequent serial fecal corticoid measures from rats reflect circadian and ovarian corticosterone rhythms. Journal of Endocrinology, 184, 153-163.

Chesler, E. J., Wilson, S. G., Lariviere, W. R., Rodriguez Zas, S. L., \& Mogil, J. S. (2002). Identification and ranking of genetic and laboratory environment factors influencing a behavioral trait, thermal nociception, via computational analysis of a large data archive. Neuroscience and Biobehavioral Reviews, 26, 907-923.

Clement, J. G. (1994). Chromodacryorrhea in rats-absence following soman poisoning. Toxicology and Applied Pharmacology, 124, 52-58.

Colegrave, N. \& Ruxton, G. D. (2003). Confidence intervals are a more useful complement to nonsignificant tests than are power calculations. Behavioral Ecology, 14, 446-447.

Crabbe, J. C., Wahlsten, D. \& Dudek, B. C. (1999). Genetics of mouse behavior: Interactions with laboratory environment. Science, 284, 1670-1672.

D’Angio, M., Serrano, A., Rivy, J. P. \& Scatton, B. (1987). Tailpinch stress increases extracellular DOPAC levels (as measured by in vivo voltammetry) in the rat nucleus accumbens but not frontal cortex: Antagonism by diazepam and zolpidem. Brain Research, 409, 169-174.

Denenberg, V. H. \& Karas, G. G. (1959). Effects of differential infantile handling upon weight gain and mortality in the rat and mouse. Science, 130, 629-630.

Denenberg, V. H. \& Smith, S. A. (1963). Effects of infantile stimulation and age upon behavior. Journal of Comparative and Physiological Psychology, 56, 307-312.

Denenberg, V. H. \& Whimbey, A. E., (1963). Infantile stimulation and animal husbandry: A methodological study. Journal of Comparative and Physiological Psychology, 56, 877878.

DeSantis, D. T. \& Schmaltz, L. W. (1984). The mother-litter relationship in developmental rat studies: Cannibalism vs caring. Developmental Psychobiology, 17, 255-262.

Eells, J. F. (1961). Inconsistency of early handling and its effect upon emotionality in the rat. Journal of Comparative and Physiological Psychology, 54, 690-693.

Fernandez-Teruel, A., Gimenez-Llort, L., Escorihuela, R. M., Gil, L., Aguilar, R., Steimer, T., \& Tobena, A. (2002). Earlylife handling stimulation and environmental enrichment: Are some of their effects mediated by similar neural mechanisms? Pharmacology Biochemistry and Behavior, 73, 233245.

Ferré, P., Núñez, J. F., García, E., Tobeña, A., Escorihuela, R. M. \& Fernández-Teruel, A. (1995). Postnatal handling 
reduces anxiety as measured by emotionality rating and hyponeophagia tests in female rats. Pharmacology Biochemistry and Behavior, 51, 199-203.

File, S. E. (1992). Behavioural detection of anxiolytic action. In: J. M. Elliott \& D. J. Heal \& C. A. Marsden (Eds.), Experimental approaches to anxiety and depression. (pp. 2544). Chichester: Wiley.

File, S. E., Andrews, N., Wu, P. Y., Zharkovsky, A. \& Zangrossi, H. Jr. (1992). Modification of chlordiazepoxide's behavioural and neurochemical effects by handling and plus-maze experience. European Journal of Pharmacology, 218, 914.

Francis, D. D., Diorio, J., Plotsky, P. M., \& Meaney, M. J. (2002). Environmental enrichment reverses the effects of maternal separation on stress reactivity. Journal of Neuroscience, 22, 7840-7843.

Galef, B. G. Jr., Mason, J. R., Preti, G., \& Bean, N. J. (1988). Carbon disulfide: A semiochemical mediating sociallyinduced diet choice in rats. Physiology and Behavior, 42, 119-124.

Gamble, M. R. (1982). Noise and laboratory animals. Journal of the Institute of Animal Technicians, 33, 5-15.

Garner, J. P., Thogerson, C. M., Mench, J. A., \& Würbel, H. (2006). Standardization and the Red Queen: Applying methodologies from ethology, neuropsychology, and field biology to problems in high-throughput behavioral methods, Paper presented at the Proceedings of the 40th International Congress of the ISAE, Bristol.

Gray, J. A., \& McNaughton, N. (2000). The neuropsychology of anxiety: An enquiry into the functions of the septo-hippocampal system.) 2nd ed.) Oxford: Oxford University Press.

Harkness, J. E., \& Ridgway, M. D. (1980). Chromodacryorrhea in laboratory rats (Rattus norvegicus): Etiologic considerations. Laboratory Animal Science, 30, 841-844.

Hayssen, V. (1998). Effect of transatlantic transport on reproduction of agouti and nonagouti deer mice, Peromyscus maniculatus. Laboratory Animals, 32, 55-64.

Heale, V. R., Vanderwolf, C. H., \& Kavaliers, M. (1994). Components of weasel and fox odors elicit fast wave bursts in the dentate gyrus of rats. Behavioural Brain Research, 63, $159-165$.

Hilakivi Clarke L. A., Turkka, J., Lister, R. G., \& Linnoila, M. (1991). Effects of early postnatal handling on brain betaadrenoceptors and behavior in tests related to stress. Brain Research, 542, 286-292.

Hoenig, J. M., \& Heisey, D. M. (2001). The abuse of power: The pervasive fallacy of power calculations for data analysis. The American Statistician, 55, 19-24.

Hogg, S. (1996). A review of the validity and variability of the elevated plus-maze as an animal model of anxiety. Pharmacology, Biochemistry and Behavior, 54, 21-30.

Huot, R. L., Thrivikraman, K. V., Meaney, M. J., \& Plotsky, P. M. (2001). Development of adult ethanol preference and anxiety as a consequence of neonatal maternal separation in Long Evans rats and reversal with antidepressant treatment. Psychopharmacology, 158, 366-373.

Kalinichev, M., Easterling, K. W., Plotsky, P. M., \& Holtzman, S. G. (2002). Long-lasting changes in stress-induced cortico- sterone response and anxiety-like behaviors as a consequence of neonatal maternal separation in Long-Evans rats. Pharmacology Biochemistry and Behavior, 73, 131-140.

Lacey, J. C., Beynon, R. J., \& Hurst, J. L. (2007). The importance of exposure to other male scents in determining competitive behaviour among inbred male mice. Applied Animal Behaviour Science, 104, 130-142.

Landi, M. S., Kreider, J. W., Lang, C. M., \& Bullock, L. P. (1982). Effects of shipping on the immune function in mice. American Journal of Veterinary Research, 43, 1654-1657.

Lehmann, J., Pryce, C. R., Jongen-Relo, A. L., Stoehr, T., Pothuizen, H. H. J., \& Feldon, J. (2002). Comparison of maternal separation and early handling in terms of their neurobehavioral effects in aged rats. Neurobiology of Aging, 23, 457-466.

Levine, S. (1958). Noxious stimulation in infant and adult rats and consummatory behavior. Journal of Comparative and Physiological Psychology, 51, 230-233.

Macri, S., Mason, G. J., \& Wurbel, H. (2004). Dissociation in the effects of neonatal maternal separations on maternal care and the offspring's HPA- and fear responses in rats. European Journal of Neuroscience, 20, 1017-1024.

Macrì, S., \& Würbel, H. (2006). Developmental plasticity of HPA and fear responses in rats: A critical review of the maternal mediation hypothesis. Hormones and Behavior, Advanced online publication.

Mason, G., Wilson, D., Hampton, C., \& Wurbel, H. (2004). Non-invasively assessing disturbance and stress in laboratory rats by scoring chromodacryorrhoea. Alternatives to Laboratory Animals, 32 153-159.

McIntosh, J., Anisman, H., \& Merali, Z. (1999). Short- and long-periods of neonatal maternal separation differentially affect anxiety and feeding in adult rats: Gender-dependent effects. Developmental Brain Research, 113, 97-106.

Meaney, M. J., \& Aitken, D. H. (1985). The effects of early postnatal handling on hippocampal glucocorticoid receptor concentrations: Temporal parameters. Developmental Brain Research, 22, 301-304.

Meaney, M. J., Mitchell, J. B., Aitken, D. H., \& Bhatnagar, S. (1991). The effects of neonatal handling on the development of the adrenocortical response to stress: Implications for neuropathology and cognitive deficits in later life. Psychoneuroendocrinology, 16, 85-103.

Moorhouse, T. P., \& Macdonald, D. W. (2005). Indirect negative impacts of radio-collaring: Sex ratio variation in water voles. Journal of Applied Ecology, 42, 91-98.

Murray, D. L., \& Fuller, M. R. (2000). A critical review of the effects of marking on the biology of vertebrates. In: L. Boitani \& T. Fuller (Eds.), Research techniques in animal ecology. (pp. 15-46). Chichester: Columbia University Press.

Plotsky, P. M., \& Meaney, M. J. (1993). Early, postnatal experience alters hypothalamic corticotropin-releasing factor (CRF) mRNA, median eminence CRF content and stressinduced release in adult rats. Molecular Brain Research, 18, 195-200.

Plotsky, P. M., Thrivikraman, K. V., Nemeroff, C. B., Caldji, C., Sharma, S., \& Meaney, M. J. E. (2005). Long-term consequences of neonatal rearing on central corticotropin- 
releasing factor systems in adult male rat offspring. Neuropsychopharmacology, 30, 2192-2204.

Pryce, C. R., \& Feldon, J. (2003). Long-term neurobehavioural impact of the postnatal environment in rats: Manipulations, effects and mediating mechanisms. Neuroscience and Biobehavioral Reviews, 27, 57-71.

Ramos, A., Berton, O., Mormede, P., \& Chaouloff, F. (1997). A multiple-test study of anxiety-related behaviours in six inbred rat strains. Behavioural Brain Research, 85, 57-69.

Rex, A., Sondern, U., Voigt, J. P., Franck, S., \& Fink, H. (1996). Strain differences in fear-motivated behavior of rats. Pharmacology Biochemistry and Behavior, 54, 107-111.

Rex, A., Voigt, J. P., Voits, M., \& Fink, H. (1998). Pharmacological evaluation of a modified open-field test sensitive to anxiolytic drugs. Pharmacology Biochemistry and Behavior, 59, 677-683.

Russell, P. A. (1971). "Infantile stimulation" in rodents: A consideration of possible mechanisms. Psychological Bulletin, 75, 192-202.

Santos, R., \& Carlini, E. A. (1988). Red lacrimal secretion (chromodacryorrhea) induced by cholinergic drugs in rats subjected to the watertank technique. Pharmacology, 36, 6168.

Severino, G. S., Fossati, I. A. M., Padoin, M. J., Gomes, C. M., Trevizan, L., Sanvitto, G. L., Franci, C. R., Anselmo-Franci, J. A., \& Lucion, A. B. (2004). Effects of neonatal handling on the behavior and prolactin stress response in male and female rats at various ages and estrous cycle phases of females. Physiology and Behavior, 81, 489-498.

Vallee, M., Mayo, W., Dellu, F., Le Moal, M., Simon, H., \& Maccari, S. (1997). Prenatal stress induces high anxiety and postnatal handling induces low anxiety in adult offspring:
Correlation with stress-induced corticosterone secretion. Journal of Neuroscience, 17, 2626-2636.

Van Loo, P. L. P., Kruitwagen, C. L. J. J., Van Zutphen, L. F. M., Koolhaas, J. M., \& Baumans, V. (2000). Modulation of aggression in male mice: Influence of cage cleaning regime and scent marks. Animal Welfare, 9, 281-295.

Võikar, V., Polus, A., Vasar, E., \& Rauvala, H. (2005). Longterm individual housing in $\mathrm{C} 57 \mathrm{BL} / 6 \mathrm{~J}$ and $\mathrm{DBA} / 2$ mice: Assessment of behavioral consequences. Genes Brain and Behavior, 4, 240-252.

von Hoersten, S., Dimitrijevic, M., Markovic, B. M., \& Jankovic, B. D. (1993). Effect of early experience on behavior and immune response in the rat. Physiology and Behavior, 54, 931940.

Wall, P. M. (2001). Methodological and conceptual issues in the use of the elevated plus-maze as a psychological measurement instrument of animal anxiety-like behavior. Neuroscience and Biobehavioral Reviews, 25, 272286.

Wang, L. (2005). A primer on rodent identification methods. Laboratory Animal, 34, 64-67.

Weinberg, J., Smotherman, W. P., \& Levine, S. (1978). Early handling effects on neophobia and conditioned taste aversion. Physiology and Behavior, 20, 589-596.

Wurbel, H. (2000). Behaviour and the standardization fallacy. Nature Genetics, 26, 263.

Wurbel, H. (2002). Behavioral phenotyping enhanced beyond (environmental) standardization. Genes Brain and Behavior, 1,3 .

Zibrowski, E. M., \& Vanderwolf, C. H. (1997). Oscillatory fast wave activity in the rat pyriform cortex: Relations to olfaction and behavior. Brain Research, 766, 39-349. 